\title{
EXTRATOS DE Brunfelsia uniflora NA GERMINAÇÃO DE SEMENTES DE Crotalaria spectabilis
}

\author{
Elizeu Junior da Silva ${ }^{1}$ \\ Samuel Pereira Custódio ${ }^{2}$ \\ Simone de Melo Santana Gomes ${ }^{3}$ \\ Victor Cesar Sartori Andre ${ }^{4}$ \\ David Maran dos Santos ${ }^{5}$ \\ Raiane Pereira Schwengber ${ }^{6}$ \\ Zilda Cristiani Gazim?
}

SILVA, E. J. da; CUSTÓDIO, S. P.; GOMES, S. de M. S.; ANDRE, V. C. S.; SANTOS, D. M. dos; SCHWENGBER, R. P.; GAZIM, Z. C. Extratos de Brunfelsia uniflora na germinação de sementes de Crotalaria spectabilis. Arq. Ciênc. Vet. Zool. UNIPAR, Umuarama, v. 19, n. 4, p. 253-257, out./dez. 2016.

RESUMO: As sementes do gênero Crotalaria L., de um modo geral, apresentam dificuldades no processo germinativo. O objetivo da pesquisa foi avaliar a eficiência do extrato bruto de Brunfelsia uniflora na germinação de Crotalaria spectabilis. O delineamento foi inteiramente casualizado, com quatro tratamentos e quatro repetições. Os tratamentos foram extratos das folhas e flores de B. uniflora nas concentrações $25,50,100 \%$ e tratamento testemunha ( $0 \%)$. O experimento foi conduzido no laboratório de Fitopatologia da Universidade Paranaense - UNIPAR, Umuarama - PR. As folhas e frutos coletados foram expostos à secagem natural e posteriormente submetidos ao processo de maceração dinâmica com esgotamento e rota evaporação do etanol, obtendo o extrato bruto. As sementes foram expostas as diluições do extratos durante dois minutos, na sequência, foram dispostas para a secagem e distribuídas em rolo papel e armazenados na B.O.D., na temperatura de $26^{\circ} \mathrm{C}$. Na contagem, aos 4 e 10 dias, determinou-se a porcentagem de plântulas normais e anormais, sementes duras e dormentes. Os extratos brutos da folha e flor de B. uniflora não ocasionaram diferenças estatísticas significativas para as variáveis analisadas, demonstrando ausência de efeito antagônico.

PALAVRAS-CHAVE: Adubos verdes. Manacá. Plantas antagonistas. Tratamento de sementes.

\section{Brunfelsia uniflora EXTRACTS IN THE GERMINATION OF Crotalaria spectabilis SEEDS}

\begin{abstract}
Seeds of the Crotalaria L. genus present difficulties in the germination process. The purpose of this study was to evaluate the efficiency of crute extract of Brunfelsia uniflora in the germination of Crotalaria spectabilis. The experimental design was completely randomized, with four treatments and four replications. The treatments consisted in $B$. uniflora leaf and flower extract at concentrations of $25,50,100 \%$ and one control treatment $(0 \%)$. The experiment was performed in the Laboratory of Phytopathology at Universidade Paranaense - UNIPAR, Umuarama - PR. The leaves and fruits collected were exposed to natural drying and then submitted to a dynamic maceration process with depletion and ethanol rotavaporation in order to obtain the crude extract. The seeds were exposed to the extract dilutions for two minutes, and then were arranged for drying, distributed on paper roll and stored in the B.O.D. at the temperature of $26^{\circ} \mathrm{C}$. At days 4 and 10 , the percentage of normal and abnormal seedlings, hard and dormant seeds were determined. The crude extracts of $B$. uniflora leaves and flowers of did not cause significant statistical differences for the variables analyzed, demonstrating the absence of an antagonistic effect. KEYWORDS: Antagonists plants. Green fertilizers. Manaca. Seed treatment.
\end{abstract}

\section{EXTRACTOS DE Brunfelsia uniflora EN GERMINACIÓN DE SEMILLAS Crotalaria spectabilis}

RESUMEN: Las semillas del género Crotalaria L., en general, presentan dificultades en el proceso de germinación. El objetivo de la investigación ha sido evaluar la eficacia del extracto crudo Brunfelsia uniflora en la germinación de Crotalaria spectabilis. El diseño fue completamente al azar, con cuatro tratamientos y cuatro repeticiones. Los tratamientos fueron los extractos de las hojas y flores de B. uniflora a concentraciones de 25,50 y $100 \%$, y tratamiento testigo ( $0 \%)$. El experimento se llevó a cabo en el Laboratorio de Fitopatología de la Universidad Paranaense - UNIPAR, Umuarama - PR. Las hojas y frutos recogidos fueron expuestos a secado natural y después sometidos al proceso de maceración dinámica con agotamiento y ruta de la evaporación del etanol, obteniendo el extracto crudo. Las semillas fueron expuestas a las diluciones de los extractos durante dos minutos, enseguida, fueron dispuestas en rollo de papel y almacenadas en B.O.D., a temperatura de $26^{\circ} \mathrm{C}$. En el recuento, al $4^{\circ}$ y $10^{\circ}$ día, se determinó el porcentaje de plántulas normales y anormales, semillas duras y latentes. Los extractos crudos de hojas y flor de $B$. uniflora no resultaron en diferencias estadísticamente significativas para las variables

DOI: https://doi.org/10.25110/arqvet.v19i4.2016.6104

${ }^{1}$ Graduando em Engenharia Agronômica, Universidade Paranaense, Umuarama, PR, Brasil. E-mail: elizeu-junior17@hotmail.com ${ }^{2}$ Graduando em Engenharia Agronômica, Universidade Paranaense, Umuarama, PR, Brasil. E-mail: samuel.mogi@gmail.com

${ }^{3}$ Programa de Pós-graduação em Biotecnologia Aplicada à Agricultura, Universidade Paranaense, Umuarama, PR, Brasil. E-mail: simonemelo@unipar.br ${ }^{4}$ Graduando em Engenharia Agronômica, Universidade Paranaense, Umuarama, PR, Brasil. E-mail:c andre.1@hotmail.com

${ }^{5}$ Graduando em Engenharia Agronômica, Universidade Paranaense, Umuarama, PR, Brasil. E-mail: davimaramdossantos@hotmail.com

${ }^{6}$ Graduando em Engenharia Agronômica, Universidade Paranaense, Umuarama, PR, Brasil. E-mail: raiane schwengber@hotmail.com

${ }^{7}$ Programa de Pós-graduação em Biotecnologia Aplicada à Agricultura, Universidade Paranaense, Umuarama, PR, Brasil. E-mail: cristianigazim@unipar.br 
analizadas, demostrando ausencia de efecto antagónico.

PALABRAS CLAVE: Abonos verdes. Brunfelsia uniflora. Plantas antagónicas. Tratamiento de semillas.

\section{Introdução}

O gênero Brunfelsia L. (Solanaceae) é constituído por arbustos que compreendem, aproximadamente, 40 espécies nativas da Bacia Amazônica (BERTRAND; COMTE; PIOLA, 2006). A espécie Brunfelsia uniflora (Pohl) D. Don é popularmente conhecida como manacá, manacá-açu, manacá-da-serra (PIO CORRÊA, 1974; BACKES; NARDINO, 2003), manacá-de-cheiro, primavera (LONGHI, 1995). Segundo Martins (2009), B. uniflora possui folhas simples, quase glabras, de quatro a sete centímetros de comprimento, com flores grandes, tubulosas, inicialmente brancas e, violetas após a fecundação, solitárias e muito perfumadas. Seus frutos são do tipo baga oblonga ou globosa (LORENZI; MATOS, 2002).

Segundo Schineider (2015), são poucas as informações que caracterizam a composição química das espécies de Brunfelsia. Estudos fitoquímicos desenvolvidos por Martins et al. (2009) evidenciaram classes de metabólitos secundários como saponinas esteroidais, ciclopropenoides, alcaloides, cumarinas e ácido clorogênico presentes de B. uniflora. As aplicações farmacêuticas (SHULTES, 1979) e a atividade antibacteriana do extrato bruto (EB) de Brunfelsia já foram comprovadas (KLOUCEK et al., 2005; QUEIROZ; BRIGHENTE, 2009).

Leguminosa anual de crescimento inicial lento (CARVALHO et al., 2003) a C. spectabilis Roth (Fabaceae) é considerada uma planta subarbustiva de porte mediano $(0,60$ a $1,50 \mathrm{~m})$, com raiz pivotante profunda, capaz de romper camadas compactadas do solo (BARRETO; FERNANDES, 2001). Promove uma maior exploração do perfil do solo (sistema radicular), maior ciclagem de nutrientes no solo (DINIZ et al., 2014), fixação de biológica de nitrogênio (WUTKE; CALEGARI; WILDNER, 2014), além de formação de palhada para o sistema de plantio direto (BALESDENT; CHENU; BALABANE, 2000), visando um manejo conservacionista. Diminuem os problemas de erosão e ocasionam melhorias na qualidade e fertilidade do solo (MALAVOLTA; GOMES; ALCARDE, 2000) podendo ainda atuar no impedimento da multiplicação de nematoides, como planta antagonista (FERRAZ et al., 2010; SANTANA et al., 2012; SANTANA-GOMES et al., 2014) e até mesmo na produção de compostos nematotóxicos (WANG; SIPES; SCHMITT, 2002).

A aplicação de procedimentos rápidos e confiáveis que possam assegurar a germinação de sementes é fundamental para a tomada de decisões dentro do processo produtivo. Entre as culturas que apresentaram acréscimo na área cultivada visando à rotação de culturas em áreas semeadas com soja, milho, algodão, a crotalária (C. spectabilis) tem se destacado (BRAZ, 2016). Segundo Callegari et al. (1993), embora a crotalária apresente crescimento determinado, o crescimento e o desenvolvimento de sementes é desuniforme.

A utilização de sementes vigorosas é algo essencial para garantir adequadas populações de plantas em diferentes condições de campo, desde a emergência até a obtenção da produtividade desejada (TEKRONY; EGLI, 1991; ROSSI, 2012). Além das diversas formas de uso, os extratos vegetais vêm sendo aplicados em outras espécies vegetais, com diversas finalidades, entre elas assegurar a germinação (DIAS et al., 2011).

Com base nisso, o presente trabalho teve por objetivo avaliar a germinação de sementes de $C$. spectabilis submetidas ao extrato bruto (EB) das folhas e flores de $B$. uniflora.

\section{Material e Métodos}

O experimento foi conduzido no Laboratório de Fitopatologia da Universidade Paranaense - UNIPAR, Campus Tiradentes (III), em Umuarama, PR. O delineamento experimental utilizado foi inteiramente casualizado, com quatro tratamentos e quatro repetições, de 100 sementes. Os tratamentos se constituíram em diluições dos EB em água com adição do solvente tween, em 25, 50 e 100\%, sendo $0 \%$ o tratamento testemunha (água pura).

Para obtenção dos extratos, as folhas e flores de manacá foram coletadas entre sete e nove horas da manhã e expostas à secagem em ambiente natural, por aproximadamente sete dias, sendo, na sequência, pulverizadas. O material foi submetido ao processo de maceração dinâmica com esgotamento do solvente (etanol 96 GL). De acordo com Nascimento (2006) e Bayoub et al. (2010) muitos dos compostos em plantas são aromáticos ou saturados de moléculas orgânicas, tornando o etanol um solvente ideal. Na sequência, realizou-se a rotaevaporação (Rota evaporador TE-210) do solvente, obtendo o EB. Para o teste, utilizaram-se sementes de C. spectabilis.

As sementes foram imersas nas diluições dos extratos durante dois minutos, sendo posteriormente distribuídas em papel para secagem e, seguidas da distribuição em rolos de papel germitest e da adição de três vezes o peso do substrato em $\mathrm{mL} / \mathrm{g}$ de água. Esses foram armazenados em estufa incubadora tipo B.O.D., em temperatura de $26^{\circ} \mathrm{C}$ com 12 horas de luz. As contagens foram realizadas aos quatro e 10 dias após a instalação do experimento. A determinação da porcentagem de germinação foi feita com base na classificação das estruturas essenciais das plântulas, conforme Brasil (2009), onde foram classificadas as plântulas normais, anormais, sementes dormentes, duras e mortas. Os dados foram transformados por $\sqrt{\mathrm{x}+1}$ e submetidos à análise de variância a $5 \%$ de probabilidade no programa SISVAR (FERREIRA, 2008).

\section{Resultados}

De acordo com as Tabelas 1 e 2, o EB das folhas e flores de $B$. uniflora não influenciaram significativamente na germinação de sementes de C. spectabilis. Todavia, o extrato das folhas na concentração de $50 \%$, apresentou os melhores resultados numéricos em relação às plântulas normais, anormais, sementes duras e sementes mortas quando comparadas aos demais tratamentos. Contudo, estatisticamente, sem efeito alelopático. 
Tabela 1: Porcentagem de germinação de sementes de $C$. spectabilis tratadas com diferentes diluições do EB das folhas de B. uniflora

\begin{tabular}{|c|c|c|c|c|c|}
\hline \multirow{2}{*}{ DILUIÇÃO (\%) } & \multicolumn{2}{|c|}{ PLÂNTULAS } & \multicolumn{3}{|c|}{ SEMENTES } \\
\hline & NORMAIS & ANORMAIS & DURAS & MORTAS & DORMENTES \\
\hline & & & $\%$ & & \\
\hline $\mathbf{0}$ & 80 & 2 & 12 & 6 & 0 \\
\hline 25 & 84 & 1 & 11 & 4 & 0 \\
\hline 50 & 87 & 1 & 9 & 3 & 0 \\
\hline 100 & 83 & 1 & 10 & 5 & 0 \\
\hline CV (\%) & 2,35 & 24,83 & 10,14 & 23,48 & 17,13 \\
\hline $\mathbf{F}$ & $0,13 \mathrm{~ns}$ & $0,98 \mathrm{~ns}$ & $0,26 \mathrm{~ns}$ & $0,25 \mathrm{~ns}$ & $0,52 \mathrm{~ns}$ \\
\hline
\end{tabular}

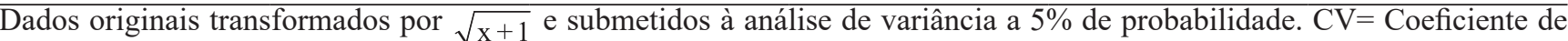
variação; ns= não significativo

Tabela 2: Porcentagem de germinação de sementes de C. spectabilis tratadas com diferentes diluições do EB das flores de B. uniflora

\begin{tabular}{cccccc}
\hline \multirow{2}{*}{ DILUIÇÃO (\%) } & \multicolumn{2}{c}{ PLÂNTULAS } & \multicolumn{3}{c}{ SEMENTES } \\
\cline { 2 - 6 } & NORMAIS & ANORMAIS & DURAS & MORTAS & DORMENTES \\
\hline $\mathbf{0}$ & 83 & & $\%$ & 5 & 1 \\
$\mathbf{2 5}$ & 83 & 1 & 10 & 5 & 0 \\
$\mathbf{5 0}$ & 79 & 2 & 10 & 7 & 1 \\
$\mathbf{1 0 0}$ & 80 & 2 & 11 & 5 & 1 \\
\hline $\mathbf{C V}(\%)$ & 2,83 & 3 & 15,56 & 13,32 & 24,15 \\
\hline $\mathbf{F}$ & $0,41 \mathrm{~ns}$ & $0,62 \mathrm{~ns}$ & $0,66 \mathrm{~ns}$ & $0,04 \mathrm{~ns}$ & $0,43 \mathrm{~ns}$
\end{tabular}

$\overline{\text { Dados originais transformados por } \sqrt{\mathrm{x}+1}}$ e submetidos à análise de variância a 5\% de probabilidade. CV $=$ Coeficiente de variação; ns= não significativo.

\section{Discussão}

Conforme os dados demonstrados nas Tabelas $1 \mathrm{e}$ 2, o extrato das folhas e flores de $B$. uniflora não apresentaram nenhuma atividade alelopática sobre a germinação das sementes de crotalária. Todavia, Queiroz e Brighente (2009) descrevem a atividade alelopática do extrato de B. uniflora a $2,0 \%$ sobre a germinação de sementes de alface (L. sativa), onde $80 \%$ das sementes tiveram sua germinação inibida pela atividade do extrato e $70 \%$ do crescimento radicular foi comprometido.

Segundo Brunner et al. (2000), o gênero Brunfelsia é rico em alcaloidesm e/ou outros constituintes ativos. Porém, mesmo na presença desses compostos, não houve efeito prejudicial ao desenvolvimento das sementes (Tabelas 1 e 2). Em um sentido amplo, qualquer efeito causado pela liberação de compostos químicos sobre a planta por um organismo doador e outro receptor recebe o nome de alelopatia, podendo ser benéfico ou não (AMORIM; REZENDE; BERGAMIM FILHO, 2011).

Dados obtidos por Oliveira et al. (2012) em um teste de germinação de sementes de espécies consideradas adubos verdes, demonstraram apenas $62 \%$ de germinação de sementes de C. spectabilis. Resultado diferente foi encontrado no presente trabalho, onde mesmo não havendo diferenças estatísticas significativas entre os tratamentos, o tratamento testemunha apresentou uma média de $82 \%$ de germinação nos dois experimentos, o que pode estar associados ao período de armazenamento de, aproximadamente, três anos.
Nesse contexto, é importante observar que C. spectabilis é uma espécie leguminosa, que apresenta tegumento duro, dificultando a entrada de água. Os tratamentos com as diluições foram realizados por um período de dois minutos, o que pode ter sido insuficiente para obtenção de resultados significativos. Ainda há poucas informações sobre a impermeabilidade do tegumento, seja devido a mudanças fisiológicas em sementes armazenadas em longos períodos ou se essa característica ocorre em função de fatores genéticos ((BEWLEY et al., 2013; MENDONÇA; PENHA, 2016). Entretanto, Egley (1979) descreveu a ocorrência de sementes de C. spectabilis impermeáveis à água.

A resistência ou tolerância aos metabólitos secundários durante a germinação é uma característica específica de cada espécie, existindo àquelas mais sensíveis como Lactuca sativa L. (alface), Lycopersicon esculentum Miller (tomate) e Cucumis sativus L. (pepino) (QUEIROZ; BRIGHENTE, 2009).

Em teste de germinação para $C$. juncea (xique-xique), Mendonça et al. (2014) obtiveram aumentos na porcentagem de germinação após o tratamento das sementes com doses até 4,8 $\mathrm{mL} \mathrm{L}^{-1}$ de extrato de Allium sativum (alho). O extrato foi obtido por meio da maceração dos dentes de alho, sendo o exsudato utilizado em diluições que variavam entre 4 e $10 \mathrm{~mL} \mathrm{~L}^{-1}$. Em um copo plástico, 60 sementes de $C$. juncea foram hidratadas em $100 \mathrm{~mL}$ das diluições do extrato de $A$. sativum em diferentes concentrações. As sementes ficaram imersas por $12 \mathrm{~h}$, à temperatura de $25^{\circ} \mathrm{C}$. Os autores também avaliaram o índice de velocidade de germinação (IVG), sen- 
do os melhores valores obtidos até a concentração de, 4,9 $\mathrm{mL} \mathrm{L} \mathrm{L}^{-1}$.

A atividade antibacteriana desse extrato pôde ser comprovada por Queiroz e Brighente (2009), que descreveram uma atividade moderada do EB de $B$. uniflora sobre a bactéria Gram-positiva Bacillus cereus. Os dados de Kloucek et al. (2005) corroboram com tais informações, uma vez que o extrato das raízes de Brunfelsia grandiflora apresentaram atividade antibacteriana frente a bactérias Gram-positivas e bactérias Gram-negativas.

Moreno-Murilo, Fajardo e Suarez (2001), utilizando o extrato etanólico das folhas de Brunfelsia pauciflora, encontraram atividade tóxica frente as larvas de Artemina salina Leach. O extrato de B. pauciflora foi obtido por meio de percolação a fresco após uma semana, sendo os resíduos dissolvidos em etanol e, posteriormente, separados por precipitação.

A confirmação da atividade antibacteriana desse extrato (KLOUCEK et al., 2005; QUEIROZ; BRIGHENTE, 2009) e a presença de diversos compostos originados do metabólito secundário faz dele um importante aliado no tratamento de sementes, principalmente para inibir o desenvolvimento de agentes patogênicos presentes no solo, que ocasionalmente possam afetar o desenvolvimento da planta e acarretar perdas de produtividade. A velocidade inicial do crescimento e rápida cobertura do solo é algo desejável, pois contribui de forma imediata com a proteção do solo, além de exercer controle contra plantas daninhas (BURLE et al., 2006).

Nesse contexto, tais resultados instigam a busca por novas informações sobre a atividade do extrato de $B$. uniflo$r a$ sobre populações de agentes fitopatogênicos, de forma a associá-lo a tratamentos de sementes, já que nas concentrações estudadas, não houve efeito antagônico na germinação das sementes, não conferindo impedimento a sua utilização.

\section{Conclusão}

O EB das folhas e flores de B. uniflora não influenciaram significativamente na porcentagem de plântulas normais, anormais, semente duras, mortas e dormentes, de $C$. spectabilis, demonstrando ausência de efeito antagônico.

\section{Referências}

AMORIM, L.; REZENDE, J. A. M.; BERGAMIM FILHO, A. Manual de fitopatologia: princípios e conceitos. 4ed. Piracicaba: Agronômica Ceres, 2011.704p.

BACKES, A.; NARDINO, M. Árvores, arbustos e algumas lianas nativas no Rio Grande do Sul. São Leopoldo: Editora Unisinos, 2003. 202p.

BALESDENT, J.; CHENU, C.; BALABANE, M. Relationship of soil organic matter dynamics to physical protection and tillage. Soil and Tillage Research, v. 53, p. 215-230, 2000.

BARRETO, A. C.; FERNANDES, M. F. Recomendações técnicas para o uso da adubação verde em solos de tabuleiros costeiros. Aracaju: Embrapa Tabuleiros Costeiros, 2001, 7 p. (Circular Técnica, 19).
BAYOUB, K.; et al. Antibacterial activities of the crude ethanol extracts of medicinal plants against Listeria monocytogenes and some other pathogenic strains. African Journal of Biotechnology, v. 9, p. 4251-4258, 2010.

BEWLEY, J. D. et al. Seeds: physiology of development, germination and dormancy. 3. ed. Springer, 2013. 392 p.

BERTRAND, C.; COMTE, G.; PIOLA, F.; Solid-phase microextraction of volatile compounds from flowers of two Brunfelsia species. Biochemical Systematics and Ecology, v. 34, p. 371-375, 2006.

BRASIL. Ministério da Agricultura, Pecuária e Abastecimento. Regras para análise de sementes. Ministério da Agricultura, Pecuária e Abastecimento. Secretaria de Defesa Agropecuária. - Brasília : Mapa/ACS, 2009. 399 p.

BRAZ, G. B. P. Crotalária: herbicidas seletivos e não seletivos e reação a nematoides. 2016. 128p. Tese (Doutorado em Agronomia: Proteção de Plantas) Universidade Estadual de Maringá, Maringá, 2016.

BRUNNER, G. et al. A novel acylated flavonol glycoside isolated from Brunfelsia grandiflora ssp. 40 Structure elucidation by gradient accelerated NMR spectroscopy at 14T. Phytochemical Analysis, v. 11, p. 29-33, 2000.

BURLE, M. L. et al. Caracterização das espécies de adubo verde. In: CARVALHO, A. M.; AMABILE, R. F. (Ed.). Cerrado: adubação verde, Embrapa Cerrados, 2006. p.71142.

CALEGARI, A. et al. Histórico. In: COSTA, M. B. B. (Coord.). Adubação verde no sul do Brasil. Rio de Janeiro: AS-PTA, 2. ed. p. 3-5, 1993.

CARVALHO, S. R. L. et al. Cinética do crescimento de leguminosas e gramíneas com alto poder relativo de penetração de raízes em solo coeso dos tabuleiros costeiros do recôncavo Baiano (Etapa I). Magistra, v. 15, p. 155$164,2003$.

DIAS, J. P. T. et al. Extrato de alho na quebra do repouso vegetativo de amoreira-preta cultivada organicamente.

Revista Trópica - Ciências Agrárias e Biológicas, v. 5, p. 23-29, 2011.

DINIZ, E. R. et al. Decomposição e mineralização do nitrogênio proveniente do adubo verde Crotalaria juncea. Científica, v. 42, n. 1, p. 51-59, 2014.

EGLEY, G. H. Seed coat impermeability and germination of showy Crotalaria (Crotalaria spectabilis) seeds. Weed Science, v. 27, n. 4, p. 355-361, 1979.

FERRAZ, S. L. G. et al. Manejo sustentável de fitonematoides. Viçosa: UFV. 2010. 306p.

FERREIRA, D. F. SISVAR: um programa para análises e ensino de estatística. Revista Symposium, v. 6, p. 36-41, 2008 . 
KLOUCEK, P. et al. Antibacterial screening of some Peruvian medicinal plants used in Caller' 1 a District. Journal of Ethnopharmacology, v. 99, p. 309-312, 2005.

LONGHI, R. A. Livro das Árvores: árvores e arvoretas do sul. Porto Alegre: L\&PM. 176p. 1995.

\section{LORENZI, H.; MATOS, F. J. A. Plantas medicinais no} Brasil, nativas e exóticas. Nova Odessa: Ed. Plantarum. 512p. 2002.

MALAVOLTA, E.; GOMES, F. P.; ALCARDE, J. C. Adubos e adubações. São Paulo: Nobel, 2000. 200p.

MARTINS, M. B. G. et al. Caracterização anatômica, química e antibacteriana de folhas de Brunfelsia uniflora (manacá) presentes na Mata Atlântica. Revista Brasileira de Farmacognosia. v. 19, p. 106-114, 2009.

MENDONÇA, G. W. et al. Extrato vegetal e fitorreguladores no condicionamento fisiológico de sementes de pimenta e crotalária. Enciclopédia Biosfera, v. 10, p. 1716-1712, 2014

MENDONÇA, V. Z.; PENHA, A. S. Influencia do período de armazenamento e quebra de dormência de guapuruvu. Tecnologia e Ciência Agropecuária, v. 10, p. 15-20, 2016.

MORENO-MURILLO, B.; FAJARDO, M. V.; SUAREZ, M. M. Cytotoxicity screening of some South American Solanaceae. Fitoterapia, v. 72, p. 680-685, 2001.

NASCIMENTO, C. O. Extração líquido - líquido da lectina da entrecasca de Crataevatapia L. utilizando micelas invertidas. 2006. 61f. Dissertação (mestrado) - Universidade Federal de Pernambuco. CCB. Ciências Biológicas. Bioquímica, 2006.

OLIVEIRA, W. P. et al. Testes de germinação em sementes de plantas utilizadas como adubo verde. In: ENCONTRO DE INICIAÇÃO CIENTÍFICA DA EMBRAPA PANTANAL E UNIVERSIDADE FEDERAL DO MATO GROSSO DO SUL, 6., 2012, Mato Grosso do Sul. Anais... Mato Grosso do Sul: UFMS, 2012, p 22.

PIO CORRÊA, M. Dicionário das plantas úteis do Brasil e das exóticas cultivadas. Rio de Janeiro: Ministério da Agricultura/Instituto Brasileiro de Desenvolvimento Florestal. 707p. 1974.

QUEIROZ, G. S.; BRIGHENTE, I. M. C. Análise de esteróides em extratos vegetais e estudo fitoquímico e biológico preliminar de Brunfelsia uniflora. Universidade Federal de Santa Catarina (UFSC). Florianópolis, 56p. 2009.

ROSSI, R. F. Vigor de sementes, população de plantas e desempenho agronômico de soja. 2012. 76p. Dissertação (Mestrado em Agronomia: Agricultura) - Faculdade de Ciências Agronômicas da UNESP, Botucatu, 2012.

SANTANA, S. M. et al. Manejo de Pratylenchus zeae por plantas antagonistas, em solos de áreas de cultivo de cana- de-açúcar. Nematropica, v. 42, p. 63-71. 2012.

SANTANA-GOMES, S. M. et al. Cropsuccession in the control of Pratylenchus brachyurus in soybean. Nematropica, v. 44, p. 200-206, 2014.

SCHNEIDER, A. L. S. Caracterização química e biológica de espécies do gênero Brunfelsia $\mathrm{L}$. navitas do Rio Grande do Sul. 95f. Dissertação (mestrado) Universidade Caxias do Sul, Programa de Pós-Graduação em Biotecnologia, 2015.

SCHULTES, R. E. Solanaceous hallucinogens and their role in the development of New World cultures. In: HAWKES, J. G.; LESTER, R. N.; SKELDING, A. D. (eds.). The Biology and Taxonomy of the Solanaceae. 1. ed. London: Academic Press, p. 137-160, 1979.

TEKRONY, D. M.; EGLI, D. B.; Relationship of seed vigor to crop yeild: a review. Crop Science, v. 31, p. 816-822, 1991.

WANG, K. H.; SIPES, B. S.; SCHMITT, D. P. Crotalaria as a cover crop for nematode magnamente: a review. Nematropica, v. 32, p. 35-57, 2002.

WUTKE, E. B.; CALEGARI, A.; WILDNER, L. P. Espécies de adubos verdes e plantas de cobertura e recomendações para seu uso. In: LIMA FILHO, O. F.; AMBROSANO, E. J.; ROSSI, F.; CARLOS, J. A. D. (Eds.) Adubação verde e plantas de cobertura no Brasil: Fundamentos e práticas. Brasília: Embrapa, 2014. p. 61167.

Recebido em: 20.11.2016 Aceito em: 26.12.2016 